\title{
A Case of Idiopathic Upper Extremitiy Deep Vein Thrombosis
}

\author{
Srikanth Evuru ${ }^{1}$, Samadi Venkata Krishna Rao ${ }^{2}$, Pradeep Botsa ${ }^{3}$ \\ ${ }^{1}$ Associate Professor of General Medicine, NRI Medical College, Chinakakani, Guntur district, AP, India \\ ${ }^{2}$ Senior Resident of General Medicine, NRI Medical College, Chinakakani, Guntur district, AP, India \\ ${ }^{3}$ Junior Resident of Critical Care Medicine, NRI Medical College, Chinakakani, Guntur district, AP, India
}

\begin{abstract}
Upper-extremity deep vein thrombosis (UEDVT) is an increasingly important clinical entity with potential for considerable morbidity. 40 year old female presented with right upper limb pain and swelling. She was diagnosed as UEDVT. She was treated with LMWH, vein patency restored and discharged.
\end{abstract}

Keywords: UEDVT, Paget-Schroetter Syndrome, coagulation disorder, fundoparinax

\section{Introduction}

Upper-extremity deep vein thrombosis (UEDVT) is an increasingly important clinical entity with potential for considerable morbidity. Pulmonary embolism (PE) is present in up to one third of patients with UEDVT.[1] Other complications, such as persistent upper-extremity pain and swelling, the superior vena cava (SVC) syndrome, and loss of vascular access, can be disabling and devastating.[2] Although once considered rare, UEDVT has become more common over the past several decades. This is directly related to the increasing use of central venous catheters for chemotherapy, bone marrow transplantation, dialysis, and parenteral nutrition. UEDVT has been reported in up to one fourth of patients with these catheters.[3] For these reasons, it is imperative that physicians understand UEDVT risk factors, diagnostic options, treatment alternatives, and prophylaxis regimens

\section{Case Presentation}

A 40 year old female presented wuth pain and swelling of right upper limb.

Present History:

Symptoms started 10 days ago, with fever, pain, redness and swelling of right upper limb.

Swelling gradually increased to present size, involving arm and fore arm. There was no other swelling invoving limbs or neck. No symptoms sugesstive of pulmonary embolism or cerebro vascular disease.

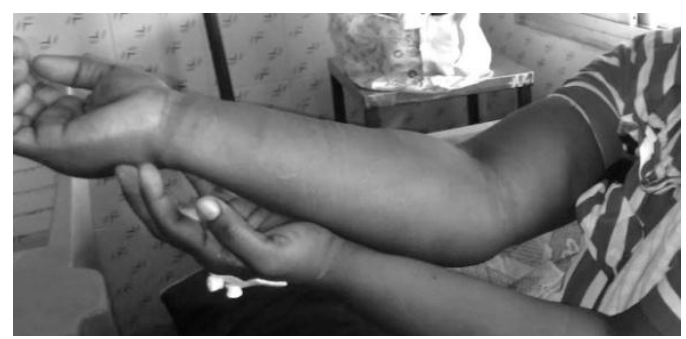

Figure 2: Right arm with UEDVT
Past History:

She was not a diabetic, hypertensive, nor does she have any chronic illness. She was not bed riden, she does not have any cancers, nor she undergoing any kind of intravenous treatment.

On examination:

Pulse rate: $89 / \mathrm{mt}$, present in all limbs, normal volume and normal rhythm.

BP: $130 / 80 \mathrm{mmHg}$,

$\mathrm{RR}-14 / \mathrm{mt}$,

Temp- 100 degees F.

On examination of affected limb, it is 1 inch circumference more in diameter compared to normal limb, both in arm and forearm. Cardivascular, respiratory, gastro intestinal and nervous system are narmal.

Investigations:

RBS- $110 \mathrm{mg} / \mathrm{dL}$,

Sr. creatinine $-1.2 \mathrm{mg} / \mathrm{dL}$,

Chest $\mathrm{x}$-ray - normal,

$\mathrm{ECG}$ - normal

CBP - normal

2Decho- normal.

Ddimer $-<12.000$ [normal $<200 \mathrm{ng} / \mathrm{ml}]$

Anti thrombin III - 0.19 [normal- 0.15-0.2 mg/ml]

Protein C - 62 [normal - $>40 \mathrm{IU} / \mathrm{dL}]$

Protein $\mathrm{S}-79$ [normal - > 63IU/dL]

Duplex ultrasound of brachial and axillary veins showed thrombus.

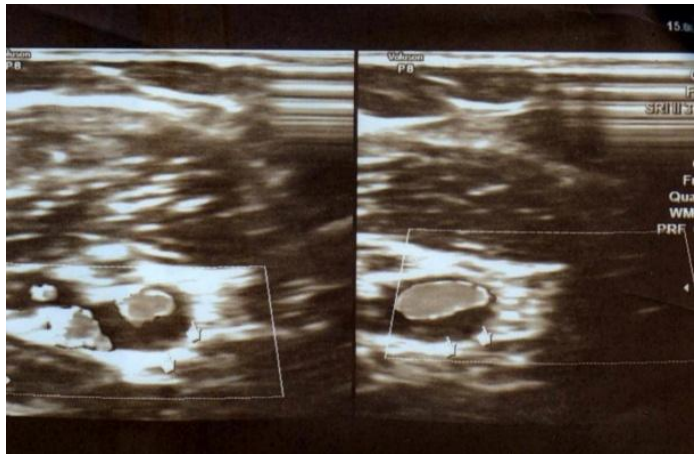

Figure 1: Duplex ultrasound showing thrombus in subclavian vein 


\section{International Journal of Science and Research (IJSR) \\ ISSN (Online): 2319-7064 \\ Index Copernicus Value (2013): 6.14 | Impact Factor (2014): 5.611}

\section{Treatment:}

She was started on fundoparinax $7.5 \mathrm{mg}$ once a day for 5 days, to continue with warfarin $2 \mathrm{mg}$ once a day. Supportive management with tight crepe bandage was applied for 5 days. She improved with the treatment in 7 days and dischared to continue warfarin for 6 months. INR was checked frequently to keep it between 2 and 3

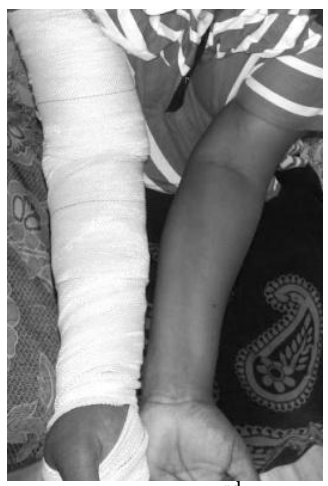

Figure 3: During treatment $3^{\text {rd }}$ day hospitalisation

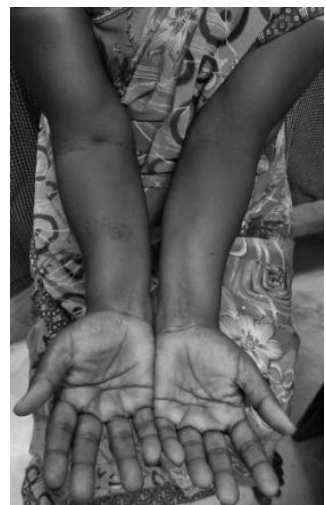

Figure 4: Normal right arm at 1 month follow up

\section{Review of Literature}

UEDVT most commonly refers to thrombosis of the axillary and/or subclavian veins. UEDVT is classified as primary or secondary on the basis of pathogenesis.

\section{Primary Thrombosis}

Primary UEDVT is a rare disorder (2 per 100000 persons per year)[4] that refers either to effort thrombosis (the socalled Paget-Schroetter Syndrome) or idiopathic UEDVT. Patients with Paget-Schroetter Syndrome develop spontaneous UEDVT, usually in their dominant arm, after strenuous activity such as rowing, wrestling, weight lifting, or baseball pitching, but are otherwise young and healthy. The heavy exertion causes microtrauma to the vessel intima and leads to activation of the coagulation cascade. Significant thrombosis may occur with repeated insults to the vein wall, especially if mechanical compression of the vessel is also present.[5]

Thoracic outlet obstruction refers to compression of the neurovascular bundle (brachial plexus, subclavian artery, and subclavian vein) as it exits the thoracic inlet. Although this disorder may initially cause intermittent, positional extrinsic vein compression, repeated trauma to the vessel can result in dense, perivascular, fibrous scar tissue formation that will compress the vein persistently.[6]
Compression of the subclavian vein typically develops in young athletes with hypertrophied muscles who do heavy lifting or completely abduct their arms. Cervical ribs, long transverse processes of the cervical spine, musculofascial bands, and clavicular or first rib anomalies are sometimes found in these patients. Therefore, cervical spine and chest plain films should be obtained in all patients undergoing evaluation for thoracic outlet syndrome.[7]

In contrast to patients with Paget-Schroetter Syndrome, patients with idiopathic UEDVT have no known trigger or obvious underlying disease. Idiopathic UEDVT may, however, be associated with occult cancer. In one study, one fourth of patients presenting with idiopathic UEDVT were diagnosed with cancer (most commonly lung cancer or lymphomas) within 1 year of follow-up. Most of these cancers were discovered during the first week of hospital admission for the venous thrombosis.[8]

The prevalence of hypercoagulable states in patients with UEDVT is uncertain because observational studies report varying results.[1-9,12] Furthermore, screening for coagulation disorders is controversial and has never been shown to be cost-effective. The yield of these tests is highest for patients presenting with idiopathic UEDVT, a family history of deep vein thrombosis (DVT), a history of recurrent, unexplained pregnancy loss, or a personal history of a prior DVT. Physicians who recommend life-long anticoagulation for protein $\mathrm{C}$, protein $\mathrm{S}$, and antithrombin III deficiencies should test for these rare causes of inherited thrombophilia. In our practice, we test for factor V Leiden, the prothrombin gene mutation, hyperhomocysteinemia, and antiphospholipid antibodies. Elevated antiphospholipid antibodies in the presence of UEDVT establish the diagnosis of the antiphospholipid antibody syndrome. We manage these patients with indefinite, intensive anticoagulation with a target international normalized ratio (INR) of 3.0 to 4.0.[13]Hyperhomocysteinemia is easily corrected with folic acid supplementation. The optimal duration of anticoagulation for a thrombotic event associated with other hypercoagulable disorders, such as factor $\mathrm{V}$ Leiden or coexisting thrombophilias, is unknown.[14]

Axillary or subclavian vein thrombosis may occasionally be completely asymptomatic. More often, though, patients complain of vague shoulder or neck discomfort and arm edema.[1] If thrombosis causes obstruction of the superior vena cava, the patient may complain of arm and facial edema, head fullness, blurred vision, vertigo, or dyspnea.[16]

Patients with thoracic outlet obstruction may have pain that radiates into the fourth and fifth digits via the medial arm and forearm, attributable to injury of the brachial plexus. Symptoms may be position dependent and worsen with hyperabduction of the shoulder or lifting. If thoracic outlet syndrome is suspected, the examiner should palpate the supraclavicular fossa for brachial plexus tenderness, inspect the hand and arm for atrophy, and perform provocative tests, such as Adson's and Wright's maneuvers. To perform the Adson test, the examiner extends the patient's arm on the affected side while the patient extends the neck and rotates the head toward the same side. Weakening of the radial

\section{Volume 4 Issue 12, December 2015}




\section{International Journal of Science and Research (IJSR) \\ ISSN (Online): 2319-7064 \\ Index Copernicus Value (2013): 6.14 | Impact Factor (2014): 5.611}

pulse with deep inspiration suggests compression of the subclavian artery. Wright's maneuver tests for reproduction of symptoms and weakening of the radial pulse when the patient's shoulder is abducted and the humerus is externally rotated.[7] Physical examination may reveal low-grade fever attributable to thrombosis. Higher fevers may suggest septic thrombophlebitis or may be related to the underlying malignancy in patients with cancer. SVC syndrome reduces venous return to the heart and, like PE, may cause sinus tachycardia. Patients with UEDVT may have mild cyanosis of the involved extremity, a palpable tender cord, [17] arm and hand edema, supraclavicular fullness, jugular venous distension, and possibly dilated cutaneous collateral veins over the chest or upper arm.[1] If a central venous catheter is present, one or multiple ports may be occluded.[16]

The signs and symptoms of UEDVT however, are nonspecific and may occur in patients with lymphedema, neoplastic compression of the blood vessels, muscle injury, or superficial vein thrombosis. Fewer than half of these symptomatic patients will have imaging evidence of an UEDVT. Therefore, it is important to confirm or exclude the diagnosis with objective testing.[1]

\section{Diagnostic imaging:}

\section{Duplex Ultrasound}

Duplex ultrasound is the initial imaging test of choice for diagnosing UEDVT because this technique is noninvasive and has high sensitivity and specificity for peripheral (jugular, distal subclavian, axillary) UEDVT.[1] Acoustic shadowing from the clavicle, however, will limit visualization of a short segment of the subclavian vein and may result in a false-negative study.[18]

\section{Contrast Venography}

Venography provides excellent characterization of venous anatomy but has several drawbacks. There may be technical difficulty in cannulating the vein in an edematous arm. The test requires an iodinated contrast agent, which may cause an allergic reaction, nephrotoxicity, or a chemical phlebitis that can worsen the preexisting thrombosis. There is little enthusiasm for using venography during pregnancy, even though iodinated contrast is rated pregnancy class $\mathrm{B}$, and radiation exposure from venography has been reported to confer minimal risk to the fetus.[19]

Despite these disadvantages, venography may be required to confirm the diagnosis of UEDVT if suspicion for clot remains high despite a negative ultrasound. Venography is also required as a prelude to interventions, such as catheterdirected thrombolysis and angioplasty, and is used to assess response to these treatments.

Magnetic Resonance Angiography

Magnetic resonance angiography (MRA) is an accurate, noninvasive method for detecting thrombus in the central thoracic veins, such as the SVC and brachiocephalic veins. MRA correlates extremely well with venography and provides more complete evaluation of central collaterals, all central veins, including contralateral vessels, and blood flow. MRA is noninvasive and may, therefore, be preferred for diagnosis, especially when contrast venography is contraindicated or impossible.[20]

\section{Treatment:}

\section{Anticoagulation}

Anticoagulation is the cornerstone of therapy. Anticoagulation helps maintain patency of venous collaterals and reduces thrombus propagation even if the clot does not completely resolve.[3] Typically, unfractionated heparin is used as a "bridge" to warfarin. Low molecular weight heparin as a bridge may be safe and effective for outpatient treatment, or for reducing the duration of hospitalization.[21] Warfarin or other anti-vitamin K agents are typically continued for a minimum of 3 months, with a goal INR of 2.0 to 3.0.[17] We recommend at least 6 months of anticoagulation therapy if a coagulation abnormality is detected.

\section{Thrombolysis}

Young and healthy UEDVT patients have significant longterm morbidity if treated only with conventional anticoagulation.[22,23] Thrombolysis restores venous patency early, minimizes damage to the vessel endothelium, and reduces the risk of long-term complications, especially the troubling post-thrombotic syndrome, which is characterized by chronic arm and hand aching and swelling.[17,22,23] In contrast, thrombolysis is rarely used for the treatment of lower extremity DVT because those patients are generally not sufficiently concerned by the potential risk of chronic leg swelling.[24]

Catheter-directed thrombolysis achieves higher rates of complete clot resolution with lower doses of medication and reduces the risk for serious bleeding compared with systemic thrombolysis. The catheter should be positioned as close to the clot as possible; otherwise, collateral circulation will carry the medication away from the thrombus. $\frac{25}{}$ Thrombolysis works best if used within several weeks of the onset of symptoms, because progressive thrombus organization will limit its effectiveness at later dates.[17,25,26]Many case series of thrombolysis in carefully selected patients have reported excellent outcomes with only minor bleeding complications, such as occasional hematomas or oozing at venipuncture or catheter sites.[25, 27-29] The thrombolysis studies are small, however, so the risks of intracranial or gastrointestinal hemorrhage may not be fully appreciated, although they probably approximate those for catheter-directed thrombolysis of lower extremity DVT

The best thrombolysis candidates are young, otherwise healthy patients with primary UEDVT, patients with symptomatic SVC syndrome, and those who require preservation of a mandatory central venous catheter. Contraindications include active bleeding, neurosurgery within the past 2 months, a history of hemorrhagic stroke, hypersensitivity to the thrombolytic agent, and surgery within the preceding 10 days. Heparin is usually given concurrently with the thrombolytic agent to prevent thrombus formation around the catheter.[17] Venipunctures,

\section{Volume 4 Issue 12, December 2015}




\section{International Journal of Science and Research (IJSR) \\ ISSN (Online): 2319-7064}

Index Copernicus Value (2013): 6.14 | Impact Factor (2014): 5.611

intramuscular injections, and invasive procedures should be minimized.

No controlled trials have compared the different thrombolytic agents. Although urokinase is an effective thrombolytic, $[25,29]$ it has been unavailable in the United States since 1999 because the Food and Drug Administration raised concerns about the safety of the manufacturing process. Subsequently, Abbott Laboratories has addressed the concerns raised by the Food and Drug Administration and hopes to reintroduce Abbokinase within the next year.

Streptokinase, an alternative thrombolytic agent, has a high rate of allergic reactions and may be ineffective if administered within months of a prior dose or streptococcal infection. Therefore, recombinant tissue plasminogen activator (rtPA) is currently the agent of choice for treating UEDVT in the United States. At our institution, catheterdirected rtPA is usually administered as a continuous infusion of 1 to $2 \mathrm{mg} / \mathrm{h}$ for at least 8 hours. Serial venography is used to assess response to treatment. Chang and colleagues[26] have reported an innovative, successful technique of delivering rtPA over 15 minutes via a pulsespray catheter lodged in the obstructing thrombus. This method may be as effective as longer infusions and may carry a lower risk of bleeding.

Percutaneous mechanical thrombectomy with devices such as the AngioJet (Possis Medical Inc) is often used in combination with thrombolytics. This procedure can rapidly extract large quantities of thrombus, thereby reducing the dose and duration of thrombolytic therapy.[33]

\section{Surgery}

Several studies have emphasized the importance of eradicating vein compression in patients with primary UEDVT to reduce the risk of recurrent thrombosis and longterm morbidity.[17,27] Therefore, after successful thrombolysis, repeat ultrasound or venography in the neutral and shoulder-abducted position can help determine whether vein compression is present.[17] Most vascular surgeons recommend early surgical correction of extrinsic vein compression, [17,27,34] which usually involves resection of part of the first rib or clavicle. ${ }^{2}$ Lysis of dense adhesions around the subclavian vein may also be required if anatomic anomalies have caused chronic, repeated trauma to the vessel. $\underline{6}$ After surgery, venography can assess residual stricture, which should be treated with balloon venoplasty; if this fails, vein stenting can be considered. Long-term patency has been documented with this multimodal approach[23,27,35,36]. Surgical thrombectomy restores venous patency but is invasive, carries the risk of general anesthesia, and may be complicated by pneumothorax and brachial plexus damage. Therefore, we reserve this technique for refractory cases.[17]

After thrombolysis, we prefer a trial of conservative therapy rather than early surgical decompression for patients with thoracic outlet syndrome. Conservative treatment, which includes a structured physical therapy program to loosen muscles compressing the subclavian vein, weight loss if obese, and nonsteroidal anti-inflammatory drugs, may obviate the need for surgery. Those with neurological symptoms due to thoracic outlet syndrome ordinarily require at least several months of physical therapy before improvement is noted.[7]

Patients with UEDVT who have contraindications to anticoagulation, such as major gastrointestinal bleeding, or patients who develop PE despite adequate anticoagulation may be candidates for SVC filter placement. SVC filters are not widely used because data regarding their safety and efficacy are sparse. There are concerns that the risks of SVC filters, including filter migration, dislodgment, fracture, and precipitation of SVC syndrome, outweigh the benefits, especially because fatal PE from UEDVT is considered rare. The very limited trials that have been completed show that SVC filters are probably safe and that they protect against clinical PE[37,38].

\section{Complication and prognosis:}

Up to one third of patients with UEDVT have PE. ${ }^{1}$ Rarely, PE secondary to UEDVT may be recurrent and fatal, despite adequate heparin therapy. Catheter removal is also a risk factor for PE. As catheters are withdrawn, fibrin sheaths may peel off the catheter, break loose from the vessel wall, and embolize[16]. The post-thrombotic syndrome, caused by venous hypertension secondary to outflow obstruction and valvular injury, varies from mild edema with little discomfort to incapacitating limb swelling with pain and ulceration. Graduated compression stockings markedly reduce the rate of the post-thrombotic syndrome in patients with lower extremity DVT.[39] Therefore, we recommend graduated compression sleeves for all symptomatic patients with acute UEDVT. Those with refractory swelling may need to use these sleeves indefinitely.

The frequency of the post-thrombotic syndrome in UEDVT patients treated only with conventional anticoagulation is uncertain, because studies are small and report conflicting results. As few as one half to as many as three fourths of these patients may develop this long-term complication[22,40,41]. Multimodal therapy that includes thrombolysis, will prevent these symptoms in the majority of patients[22,35,36,40]. Those with primary UEDVT are usually young and healthy, more active, live longer, and are not troubled by other chronic medical conditions. Therefore, they should receive more aggressive treatment, such as thrombolysis and correction of outlet obstruction, to reduce the risk of chronic venous insufficiency. Patients with secondary UEDVT are less bothered by symptoms and are often not candidates for surgery or thrombolysis, so conservative treatment with anticoagulation alone is generally recommended. These patients have very high short-term mortality rates compared with patients who have lower extremity deep vein thrombosis. Most die from underlying medical problems such as infection, cancer, or multisystem organ failure rather than from complications of the UEDVT.

Other complications include SVC syndrome, septic thrombophlebitis, thoracic duct obstruction, and brachial plexopathy. $\stackrel{2}{=}$ Loss of vascular access can be especially problematic if UEDVT prevents administration of essential medication or nutrition.

\section{Volume 4 Issue 12, December 2015}




\section{International Journal of Science and Research (IJSR) \\ ISSN (Online): 2319-7064}

Index Copernicus Value (2013): 6.14 | Impact Factor (2014): 5.611

\section{Prophylaxis}

On the basis of studies by Bern et al[43] and Boraks et al,[44] some physicians prescribe a "mini-dose" (1 mg) of warfarin daily to their cancer patients with central venous catheters to potentially reduce the risk of developing subsequent UEDVT. This low dose usually does not prolong the prothrombin time or cause clinical bleeding. Patients with poor nutrition, those receiving broad spectrum antibiotics, or those with advanced liver disease or liver metastases may not be suitable candidates for warfarin prophylaxis, because in these situations, even the tiny dose of $1 \mathrm{mg}$ may be sufficient to elevate the prothrombin time excessively.

Low molecular weight heparin is an alternative to warfarin for UEDVT prophylaxis in cancer patients with central venous catheters. Monreal and colleagues[45] showed that once daily subcutaneous administration of 2500 IU of dalteparin starting 2 hours before catheter insertion greatly reduces the frequency of UEDVT. There were no bleeding complications, even when patients received chemotherapy that caused bone marrow suppression. Low molecular weight heparin is a better choice than warfarin for prophylaxis of patients with liver dysfunction or malnutrition.

\section{Discussion}

Our patient was 40year old female, who was an ayya by profession, came with a history of swelling, redness and pain of right upper limb. Thompson RW [6] et al was on an opinion that effort on dominant limb can cause thrombosis due to repeated micro trauma to the vein, which might have lead to the thrombosis in our patient. GirolamiA [8] et al studied the frequency of cancers i.e lung in patients presenting with UEDVT, on evaluation of our patient, no cancers were present at presentation and at frequent followups at 1 year. Prandoni $P$ [1] et al stressed the importance and cost effectiveness of duplex ultrasound, simillarly in our patient the investigation was done and thrombus demonstrated. Duplex ultrasound was done at discharge too, to demonstrate the patency of the vein. Anti coagulation is the cornerstone of treatment [1], [17] and thrombolysis is preferred only in catheter induced thrombosis or in lower extremity DVT [17.23,25,27-29,32, 35]. rtPA, percutaneous thrombectomy, surgery were considered by a few collegues $[26,27,33,34]$. In our patient we went for anticoagulation therapy due to ease of administration, financial constraints.

\section{References}

[1] Prandoni P, Polistena P, Bernardi E, et al. Upperextremity deep vein thrombosis: risk factors, diagnosis and complications. Arch Intern Med.1997; 157: 57-62.

[2] Becker DM, Philbrick JT, Walker FB. Axillary and subclavian venous thrombosis: prognosis and treatment. Arch Intern Med. 1991; 151: 1934-1943.

[3] Horattas MC, Wright DJ, Fenton AH, et al. Changing concepts of deep venous thrombosis of the upper extremity: report of a series and review of the literature. Surgery. 1988; 104: 561-567.
[4] Lindblad B, Tengborn L, Bergqvist D. Deep vein thrombosis of the axillary-subclavian veins: epidemiologic data, effects of different types of treatment and late sequelae. Eur J Vasc Surg. 1988; 2: 161-165.

[5] Zell L, Kindermann W, Marschall F, et al. PagetSchroetter syndrome in sports activities: case study and literature review. Angiology. 2001; 52: 337-342.

[6] Thompson RW, Schneider PA, Nelken NA, et al. Circumferential venolysis and paraclavicular thoracic outlet decompression for "effort thrombosis" of the subclavian vein. J Vasc Surg. 1992; 16: 723-732.

[7] Parziale JR, Akelman E, Weiss AP, et al. Thoracic outlet syndrome. Am J Orthop. 2000; 29: 353-360.

[8] Girolami A, Prandoni P, Zanon E, et al. Venous thromboses of upper limbs are more frequently associated with occult cancer as compared with those of lower limbs. Blood Coag Fibrinol. 1999; 10: 455-457.

[9] Heron E, Lozinguez O, Alhenc-Gelas $M$, et al. Hypercoagulable states in primary upper-extremity deep vein thrombosis. Arch Intern Med. 2000; 160:382-386.

[10] Leebeek FW, Stadhouders NA, van Stein D, et al. Hypercoagulability states in upper-extremity deep venous thrombosis. Am J Hematol. 2001; 67: 15-19.

[11] Martinelli I, Cattaneo M, Panzeri D, et al. Risk factors for deep venous thrombosis of the upper extremities. Ann Intern Med. 1997; 126: 707-711.

[12] Ruggeri M, Castaman G, Tosetto A, et al. Low prevalence of thrombophilic coagulation defects in patients with deep vein thrombosis of the upper limbs.Blood Coagul Fibrinolysis. 1997; 8: 191-194.

[13] Khamashta MA, Cuadrado MJ, Mujic F, et al. The management of thrombosis in the antiphospholipidantibody syndrome. N Engl J Med. 1995;332: 993-997.

[14] Seligsohn U, Lubetsky A. Genetic susceptibility to venous thrombosis. N Engl J Med. 2001; 344: 12221231.

[15]Luciani A, Clement O, Halimi P, et al. Catheter-related upper extremity deep venous thrombosis in cancer patients: a prospective study based on doppler US. Radiology. 2001; 220: 655-660.

[16] Mayo DJ. Catheter-related thrombosis. J Intraven Nurs. 2001; 24: S13-S22.

[17] Hicken GJ, Ameli M. Management of subclavianaxillary vein thrombosis: a review. Can J Surg. 1998; 41: 13-25.

[18] Haire WD, Lynch TG, Lund GB, et al. Limitations of magnetic resonance imaging and ultrasound-directed (duplex) scanning in the diagnosis of subclavian vein thrombosis. J Vasc Surg. 1991; 13: 391-397.

[19] Toglia MR, Weg JG. Venous thromboembolism during pregnancy. N Engl J Med. 1996; 335: 108-114.

[20] Hartnell GG, Hughes LA, Finn JP, et al. Magnetic resonance angiography of the central chest veins: a new gold standard? Chest. 1995; 107: 1053-1057.

[21] Savage KJ, Wells PS, Schulz V, et al. Outpatient use of low molecular weight heparin (dalteparin) for the treatment of deep vein thrombosis of the upper extremity. Thromb Haemost. 1999; 82: 1008-1010.

[22] AbuRahma AF, Sadler DL, Robinson PA. Axillarysubclavian vein thrombosis: changing patterns of etiology, diagnostic, and therapeutic modalities. Am Surg. 1991; 57: 101-107. 


\section{International Journal of Science and Research (IJSR) \\ ISSN (Online): 2319-7064}

Index Copernicus Value (2013): 6.14 | Impact Factor (2014): 5.611

[23] Urschel HC, Razzuk MA. Paget-Schroetter syndrome: what is the best management? Ann Thorac Surg. 2000; 69: 1663-1669.

[24] O’Meara JJ, McNutt RA, Evans AT, et al. A decision analysis of streptokinase plus heparin as compared with heparin alone for deep-vein thrombosis. N Engl J Med. 1994; 330: 1864-1869.

[25] Fraschini G, Jadeja J, Lawson M, et al. Local infusion of urokinase for the lysis of thrombosis associated with permanent central venous catheters in cancer patients. J Clin Oncol. 1987; 5: 672-678.

[26]Chang R, Horne MK, Mayo DJ, et al. Pulse-spray treatment of subclavian and jugular venous thrombi with recombinant tissue plasminogen activator. J Vasc Interv Radiol. 1996; 7: 845-851.

[27] Machleder HI. Evaluation of a new treatment strategy for Paget-Schroetter syndrome: spontaneous thrombosis of the axillary-subclavian vein. J Vasc Surg. 1993; 17: 305-317.

[28] Beygui RE, Olcott C, Dalman RL. Subclavian vein thrombosis: outcome analysis based on etiology and modality of treatment. Ann Vasc Surg. 1997;11: 247255.

[29] Seigel EL, Jew AC, Delcore R, et al. Thrombolytic therapy for catheter-related thrombosis. Am J Surg. 1993; 166: 716-719.

[30] Bjarnason H, Kruse JR, Asinger DA, et al. Iliofemoral deep venous thrombosis: safety and efficacy outcome during 5 years of catheter-directed thrombolytic therapy. J Vasc Interv Radiol. 1997; 8: 405-418.

[31] Mewissen MW, Seabrook GR, Meissner MH. Catheterdirected thrombolysis for lower extremity deep venous thrombosis: report of a national multicenter registry. Radiology. 1999; 211: 39-49.

[32] Martin M. Results of the PHLEFI study (phlebothrombosis-fibrinolytic therapy): a prospective, multicenter study of the fate of 1498 patients receiving fibrinolytic therapy for deep vein thrombosis. Int $\mathrm{J}$ Angiology. 1998;7: 68-76.

[33] Kasirajan K, Gray B, Ouriel K. Percutaneous AngioJet thrombectomy in the management of extensive deep venous thrombosis. J Vasc Interv Radiol.2001; 12: 179185.

[34] Lee MC, Grassi CJ, Belkin M, et al. Early operative intervention after thrombolytic therapy for primary subclavian vein thrombosis: an effective treatment approach. J Vasc Surg. 1998; 27: 1101-1108.

[35] Adelman MAA, Stone DH, Riles TS, et al. A multidisciplinary approach to the treatment of PagetSchroetter syndrome. Ann Vasc Surg. 1997; 11: 149154.

[36] Kreienberg PB, Chang BB, Darling RC, et al. Longterm results in patients treated with thrombolysis, thoracic inlet decompression, and subclavian vein stenting for Paget-Schroetter syndrome. J Vasc Surg. 2001; 33: S100-S105.

[37] Ascher E, Hingorani A, Tsemekhin B, et al. Lessons learned from a 6-year clinical experience with superior vena cava Greenfield filters. J Vasc Surg.2000; 32: 881-887.

[38] Spence LD, Gironta MG, Malde H, et al. Acute upper extremity deep venous thrombosis: safety and effectiveness of superior vena caval filters. Radiology.1999; 210: 53-58.

[39] Brandjes DPM, Buller HR, Heijboer H, et al. Randomised trial of the effect of compression stockings in patients with symptomatic proximal-vein thrombosis. Lancet. 1997; 349: 759-762.

[40] AbuRahma AF, Robinson PA. Effort subclavian vein thrombosis: evolution of management. J Endovasc Ther. 2000; 7: 302-308.

[41]Heron E, Lozinguez O, Emmerich J, et al. Long-term sequelae of spontaneous axillary-subclavian venous thrombosis. Ann Intern Med. 1999;131: 510-513.

[42] Hingorani A, Ascher E, Lorenson E, et al. Upper extremity deep venous thrombosis and its impact on morbidity and mortality rates in a hospital-based population. J Vasc Surg. 1997; 26: 853-860.

[43] Bern MM, Lokich JJ, Wallach SR, et al. Very low doses of warfarin can prevent thrombosis in central venous catheters: a randomized prospective trial. Ann Intern Med. 1990; 112: 423-428.

[44] Boraks P, Seale J, Price J, et al. Prevention of central venous catheter associated thrombosis using minidose warfarin in patients with haematological malignancies. Br J Haematol. 1998; 101: 483-486.

[45] Monreal M, Alastrue A, Rull M, et al. Upper extremity deep venous thrombosis in cancer patients with venous access devices: prophylaxis with a low molecular weight heparin (fragmin). Thromb Haemost. 1996; 75: 251-253.

[46] Francis CW, Suchkova VN. Ultrasound and thrombolysis. Vasc Med. 2001;6: 181-187.

\section{Author Profile}

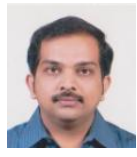

Dr. Srikanth Evuru received MBBS degree from Rangaraya Medical College, Kakinada, AP, India, M.D post graduation from rajah muttiah medical college, Chidambaram, TN, INDIA, he worked as an assistant professor and subsequently working as associate professor at NRI Medical College, Chinakakani, AP, India

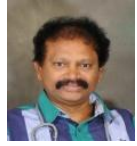

Dr. Samadi Venkata Krishna Rao recievied MBBS degree from S.V.medical college, Tirupathi, AP India, M.D post graduation from S.V. Medical College, Tirupathi, AP, INDIA, he is working as senior resident at NRI Medical College, Chinakakani, AP, India.

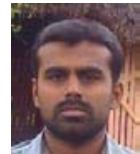

Dr. Pradeep Botsa recievied MBBS degree from Guntur medical college, Guntur, AP, India, he is working as junior resident in the department of critical care at NRI Medical College, Chinakakani, AP, India. 